\title{
L'obésité : le prochain enjeu de santé publique
}

\author{
par Christine M. Bond
}

A u cours des dernières décennies, l'espérance de vie a augmenté de façon spectaculaire dans les pays développés et bon nombre de personnes ont maintenant une meilleure qualité de vie à un âge chronologique qu'il aurait été possible d'imaginer il y a 40 ans. On a non seulement ajouté des années à la vie, mais de la qualité à ces années. Ces changements sont attribuables à une meilleure connaissance des processus morbides et de leur gestion. Les pharmacothérapies et les pharmaciens ont contribué à ces améliorations. Mais ce n'est qu'une partie de l'histoire : une bonne partie de la transformation du profil démographique provient d'une meilleure compréhension des moyens à prendre pour prévenir les maladies grâce aux approches de santé publique visant à améliorer les modes de vie. Au Royaume-Uni, la prévalence du tabagisme, autrefois de $50 \%{ }^{1}$, est aujourd'hui de $16 \%{ }^{2}$ et, dans bon nombre de sous-groupes de la population, elle est encore plus faible. Au Canada, elle est aussi à son niveau le plus bas, à environ $13 \%{ }^{3}$. Les réductions du tabagisme se sont traduites en des baisses des cas de coronaropathie et de cancer; en effet, la désaccoutumance du tabac a été étiquetée comme l'intervention en santé la plus efficace qui soit par rapport au coût. Les pharmaciens ont d'ailleurs grandement contribué à ce changement culturel.

Il reste toujours du travail à faire en ce qui concerne la désaccoutumance du tabac, mais nous disposons d'un système fondé sur des données probantes et doté des ressources adéquates que nous pouvons continuer de mettre en œuvre. Ce n'est pas le cas pour l'obésité, largement considérée comme le prochain enjeu de santé publique, un problème contre lequel les pharmaciens peuvent et doivent jouer un rôle. Un adulte sur 4 et un enfant sur 10 au Canada sont cliniquement obèses ${ }^{4}$ et dans certains groupes, notamment chez les peuples autochtones ${ }^{5}$, la prévalence est significativement plus élevée. Ainsi, environ 6 millions de Canadiens souffriraient d'obésité et pourraient avoir besoin d'une aide immédiate pour gérer et contrôler leur poids. Bien que l'obésité soit un problème moins grave au Canada qu'aux ÉtatsUnis, où plus d'un tiers des adultes ${ }^{6}$ et jusqu'à un cinquième des enfants sont obèses ${ }^{7}$, le Canada doit s'attaquer à cette potentielle bombe à retardement. Selon un rapport de 2010, les coûts directs en santé pour le surpoids et l'obésité au Canada s'élevaient à
6 milliards de dollars, environ 4,1\% de l'ensemble du budget de la santé $e^{4}$ De plus, cette évaluation ne prend pas en compte les pertes de productivité, la baisse des recettes fiscales ou les coûts psychosociaux ${ }^{4}$. En 2011, au cours d'une rencontre de haut niveau de l'Assemblée générale des Nations Unies sur la prévention et le contrôle des maladies transmissibles ${ }^{8}$, la croissance de la prévalence de l'obésité chez les nourrissons, les enfants et les adolescents a été un sujet particulier de préoccupation. On a recommandé la mise en place de la Stratégie mondiale pour l'alimentation, l'exercice physique et la santé de l'Organisation mondiale de la Santé, notamment (le cas échéant) les politiques et les mesures encourageant une alimentation saine et une augmentation de l'exercice physique. Les enfants obèses font face à des problèmes de santé immédiats, atteignent moins d'objectifs éducatifs courants et ont une moins bonne qualité de vie. De plus, d'après des données probantes, un enfant obèse deviendra un adulte obèse qui risquera alors de souffrir du diabète de type 2 , de cardiopathies, d'accidents vasculaires cérébraux, de démences, de maladies rénales et de certains cancers.

Des initiatives globales publiques, privées et non gouvernementales sur la prévention et le traitement de l'obésité doivent être mises en place d'urgence. Les pharmaciens participent déjà à la prestation de programmes de gestion du poids 9 . Toutefois, il n'y a pas suffisamment de données probantes pour démontrer l'efficacité des pharmaciens à cet égard ${ }^{10}$ et leur contribution à la lutte contre l'obésité doit être étudiée de plus près ${ }^{11}$. Par contre, des données probantes indiquent que le dépistage mené par les médecins de famille et les brefs conseils de motivation sont efficaces $^{12}$. Il n'y a aucune raison qui devrait empêcher les pharmaciens travaillant en milieu communautaire de prodiguer un service structuré semblable, accordant la priorité aux patients en excès de poids atteints d'affections aggravées par l'obésité (par exemple, le diabète de type 2 et l'arthrose). Tout comme c'est le cas dans les programmes de désaccoutumance du tabac, les pharmaciens peuvent réaliser efficacement des interventions pour modifier le mode de vie, ce qui permettrait aux médecins de se concentrer sur les aspects cliniques plus complexes de l'obésité. Cependant, les changements au mode de vie, parmi lesquels on compte les régimes et l'exercice, ne sont pas nécessairement 
suffisants. Lorsqu'un patient souffre d'obésité grave ou morbide, des approches à plusieurs volets sont de rigueur. Comparativement à la désaccoutumance du tabac, là ou des approches comportementales complètent généralement une pharmacothérapie, la gestion de l'obésité chez l'adulte peut nécessiter des approches pharmacologiques, chirurgicales et comportementales. L'orlistat, qui réduit l'absorption des graisses alimentaires par le corps, est maintenant largement utilisé avec de bons résultats et, mis à part des effets indésirables mineurs, il est généralement bien toléré. Bien qu'il s'agisse surtout d'un médicament d'ordonnance, il est aussi en vente libre dans bon nombre de pays, dont les États-Unis, et est disponible sur le Web au Canada. D'autres traitements pour l'obésité agissent sur le système nerveux central en réduisant l'appétit (par exemple, la lorcasérine ou la phentermine) et en provoquant la satiété. De tels médicaments sont plus à risque de provoquer des effets indésirables, d'intervenir dans d'importantes interactions médicamenteuses ou d'être contre-indiqués, par exemple, pour les patients atteints de cardiopathie. Les pharmaciens ont donc des occasions de donner des conseils sur le traitement le plus pertinent, d'attirer l'attention sur les potentielles interactions médicamenteuses et de repérer les patients présentant des contreindications. Qui plus est, ils peuvent mettre en garde contre les dangers des régimes miracles et les risques potentiels des achats en ligne. Ainsi, tous les pharmaciens pourraient aider les gens à gérer leur poids.

Il ne s'agit pas de savoir si les pharmaciens auraient un rôle à jouer dans la gestion de l'épidémie d'obésité, mais plutôt s'ils peuvent accomplir un tel rôle. Les pharmaciens sont-ils en mesure d'adapter les techniques de changement de comportement employées pour la désaccoutumance du tabac à un contexte différent et potentiellement plus délicat? Le public acceptera-t-il de telles interventions du pharmacien? Les pharmaciens possèdent-ils les connaissances nécessaires leur permettant d'offrir des conseils à leurs collègues en médecine sur la meilleure approche pharmacologique à adopter? Les décideurs et les gestionnaires considéreront-ils les pharmaciens comme des membres de l'équipe de spécialistes? Il s'agit là de questions et d'enjeux centraux que les éducateurs, les chercheurs et les gestionnaires en santé doivent aborder.

Enfin, tout cela se déroule sur fond d'environnement obésogène et tout en sachant que certaines personnes sont génétiquement prédisposées à l'obésité. La malbouffe et les boissons gazeuses sont consommées quotidiennement par bien des gens, et des obstacles (la météo, par exemple) et des éléments dissuasifs (le manque de temps entre autres) à l'activité physique comme la marche se dressent dans leur quotidien. Or, bien qu'une prévention précoce, incluant des changements à l'environnement et une évolution des normes culturelles, représente la solution à long terme, il y a un problème immédiat à prendre en main. Une approche systémique globale est maintenant nécessaire pour répondre aux besoins actuels de la population et il est important que tous les pharmaciens participent aux côtés d'autres professionnels de la santé.

[Traduction par l'éditeur]

\section{Références}

1. Adult smoking habits in the UK: 2016 [bulletin statistique]. Newport (Pays de Galles) : Office for National Statistics; 2017. Publié au : https:// www.ons.gov.uk/peoplepopulationandcommunity/healthandsocialcare/healt handlifeexpectancies/bulletins/adultsmokinghabitsingreatbritain/2016. Consulté le 18 août 2017.

2. Smoking rate in UK falls to second-lowest in Europe. The Guardian 2017 Jun 15. Publié au : https://www.theguardian.com/society/2017/jun/15/ smoking-rate-in-uk-falls-to-second-lowest-in-europe. Consulté le 18 août 2017.

3. Tobacco use in Canada: highlights. Waterloo $(\mathrm{ON})$ : University of Waterloo, Propel Centre for Population Health Impact; 2017. Publié au : https:// uwaterloo.ca/tobacco-use-canada/highlights. Consulté le 18 août 2017.

4. Obesity in Canada [page d'accueil sur Internet]. Edmonton (AB) : Canadian Obesity Network. Publié au : www.obesitynetwork.ca/obesity-in-canada. Consulté le 18 août 2017.

5. Obésité au Canada - Prévalence de l'obésité au sein des populations autochtones. Ottawa (ON) : Agence de la santé publique du Canada; [modifié le 23 juin 2011]. Publié au : https://www.canada.ca/fr/santepublique/services/promotion-sante/modes-vie-sains/obesite-canada/ prevelance-obesite-populations-autochtones.html. Consulté le 8 août 2017.

6. Overweight and obesity: adult obesity facts. Atlanta (GA) : Centers for Disease Control and Prevention. Publié au : https://www.cdc.gov/obesity/ data/adult.html. Consulté le 18 août 2017.

7. The state of childhood obesity. Dans: The state of obesity: better policies for a healthier America [site Web]. Washington (DC): Trust for America's Health and Robert Wood Johnson Foundation; [mis à jour en août 2017]. Publié au : www.stateofobesity.org. Consulté le 18 août 2017.

8. Points 24 et $43 \mathrm{~d}$. Dans : Political declaration of the High-level Meeting of the General Assembly on the Prevention and Control of Non-communicable Diseases [résolution préliminaire soumise au président de l'Assemblée générale]. New York (NY) : Assemblée générale des Nations Unies; 2011. Publié au : www.un.org/ga/search/view_doc.asp?symbol=A/66/L.1. Consulté le 18 août 2017.

9. Murphy AL, Gardner DM. A scoping review of weight bias by community pharmacists towards people with obesity and mental illness. Can Pharm J. 2016;149(4):226-35.

10. Gordon J, Watson M, Avenell A. Lightening the load? A systematic review of community pharmacy-based weight management interventions. Obes Rev. 2011;12(11):897-911.

11. McVey GL, Walker KS, Beyers J, Harrison HL, Simkins SW, Russell-Mayhew $S$. Integrating weight bias awareness and mental health promotion into obesity prevention delivery: a public health pilot study. Prev Chronic Dis. 4 avril 2013;10:E46.

12. Aveyard P, Lewis A, Tearne S, Hood K, Christian-Brown A, Adab P, et al. Screening and brief intervention for obesity in primary care: a parallel, twoarm, randomised trial. Lancet. 2016;388(10059):2492-500.

Christine M. Bond, B. Pharm., Ph. D., M. Ed., est professeure émérite au Centre of Academic Primary Care de I'Université d'Aberdeen, Foresterhill, Aberdeen, Écosse. Elle est également rédactrice adjointe du Journal canadien de la pharmacie hospitalière.

Intérêts concurrents : Aucun déclaré

\section{Adresse de correspondance:}

Dre Christine M. Bond

Pharmacy

Centre of Academic Primary Care

Polwarth Building West Block, Room 1.123

Foresterhill, Aberdeen AB25 2ZD

Scotland

Courriel : c.m.bond@abdn.ac.uk 